\title{
Long non-coding RNA TP73-AS1 accelerates the progression and cisplatin resistance of non-small cell lung cancer by upregulating the expression of TRIM29 via competitively targeting microRNA-34a-5p
}

\author{
SHUNXIANG LUO, MING SHEN, HUI CHEN, WEIWEI LI and CONG CHEN \\ Department of Oncology, The First People's Hospital of Tianmen, Tianmen, Hubei 431700, P.R. China
}

Received October 14, 2019; Accepted May 29, 2020

DOI: $10.3892 / \mathrm{mmr} .2020 .11473$

\begin{abstract}
Non-small cell lung cancer (NSCLC) is a leading subtype of lung cancer, with high mortality rates. Recently, long non-coding RNAs (lncRNAs) have been associated with NSCLC. The present study aimed to examine the role of the TP73 antisense RNA 1 (TP73-AS1) lncRNA in NSCLC. TP73-AS1 and microRNA(miR)-34a-5p expression levels were measured using reverse transcription-quantitative PCR (RT-qPCR) and chromogenic in situ hybridization (CISH). Cell proliferation, apoptosis, migration and invasion was determined using Cell Counting Kit-8 (CCK-8), flow cytometry, Transwell and Matrigel assays, respectively. The median inhibitory concentration $\left(\mathrm{IC}_{50}\right)$ value of cisplatin (cis-diamminedichloroplatinum; DDP) was assessed using a CCK-8 assay. The interaction between miR-34a-5p and TP73-AS1 or tripartite motif-containing 29 (TRIM29) was predicted using microRNA. org and Starbase, then verified using a dual-luciferase reporter assay. The expression of TRIM29 was quantified at the mRNA and protein level using RT-qPCR and western blot analysis, respectively. TP73-AS1 was significantly upregulated, while miR-34a-5p was downregulated in NSCLC tissues and cells. Functionally, TP73-AS1 knockdown inhibited proliferation, migration, invasion and DDP resistance, whilst inducing apoptosis in NSCLC cells. miR-34a-5p was identified as a target for TP73-AS1, and its inhibition reversed the effects of TP73-AS1 knockdown on NSCLC cells. In addition, TRIM29 was targeted by miR-34a-5p, and its overexpression reversed the effects of miR-34a-5p. Moreover, TP73-AS1 acted as a molecular sponge for miR-34a-5p, increasing the expression of TRIM29. In
\end{abstract}

Correspondence to: Mr. Cong Chen, Department of Oncology, The First People's Hospital of Tianmen, 1 East Renmin Avenue, Tianmen, Hubei 431700, P.R. China

E-mail: qbdruq@163.com

Key words: tumor protein 73 antisense RNA 1, miR-34a-5p, tripartite motif-containing 29 , cisplatin resistance, non-small cell lung cancer conclusion, TP73-AS1 contributed to proliferation, migration and DDP resistance but inhibited apoptosis of NSCLC cells by upregulating TRIM29 and sponging miR-34a-5p.

\section{Introduction}

Lung cancer remains the leading cause of cancer-associated death worldwide, and the mortality rate in 2019 was higher in males (24\%) compared with females (23\%) in 2019 in USA (1). Non-small cell lung cancer (NSCLC) is a primary form of lung cancer that accounts for $>80 \%$ of all lung cancer cases worldwide (2). NSCLC has two major subtypes based on clinical pathology, squamous cell carcinoma and adenocarcinoma (3). The pathogenesis of NSCLC is complex and poorly understood, and a previous study recorded from Brazil that the rates of recurrence (over 50\%) and mortality (69.7\%) following surgery and different lines of chemotherapy remain extremely high (4). A previous study suggested that novel targets (ROS1 and RET fusions) would benefit NSCLC treatment (5). Therefore, further research on potential therapeutic targets associated with NSCLC remains a necessity.

Long non-coding RNAs (lncRNAs) are RNA molecules $>200$ nucleotides in length with no or limited protein-coding capacity (6). IncRNAs are involved in multiple biological processes, including cell cycle, cell differentiation and myogenesis, by acting as microRNAs sponges or antisense modulators of mRNA transcripts (7). Recently, lncRNAs have been implicated in several types of cancer, including NSCLC. For example, lncRNA prostate cancer associated transcript 6 acted as an oncogene in NSCLC, contributing to tumor cell growth, migration and invasion (8). Another previous study suggested that small nucleolar RNA host gene 1 was upregulated in NSCLC and promoted tumor cell viability, proliferation and metastasis in NSCLC (9). In addition, lncRNA VPS9D1-antisense RNA 1 (AS1) was overexpressed in NSCLC, which was associated with high rates of metastasis and short survival time within $\sim 10$ years (10). Altogether, these previous studies suggested that lncRNAs played essential roles in the progression of NSCLC. Tumor protein 73 AS1 (TP73-AS1) has been found to be involved in several types of cancer $(11,12)$. However, the role of TP73-AS1 in the development of NSCLC remains unclear. 
MicroRNAs (miRNAs) are non-coding RNA molecules, 18-22 nucleotides in length (13). MiRNAs interact with downstream mRNAs, leading to mRNA degradation or translation repression. Therefore, miRNAs modulate diverse cellular processes, including cell differentiation, proliferation and development $(14,15)$. Aberrant increased or decreased expression of miRNAs has been implicated in different human diseases, particularly cancer (16). Previous studies demonstrated that miR-34a-5p acted as a tumor suppressor in various types of cancer, such as colorectal cancer (17), cervical cancer (18) and pancreatic carcinoma (19). However, the role of miR-34a-5p in lung cancer is unknown.

Tripartite motif-containing 29 (TRIM29) is a member of the TRIM protein family, which contains multiple zinc finger motifs and a leucine zipper motif (20). The TRIM29 gene is located on chromosome 11q23 and plays a role in numerous biological processes, including DNA assembly, protein repair and cancer progression $(21,22)$. Notably, TRIM29 not only serves as a tumor inhibitor, such as in invasive breast cancer (23), but also acts as an oncogene in gastric cancer (24), thyroid carcinoma (25) and bladder cancer (26). Nevertheless, the potential role played by TRIM29 in NSCLC is poorly understood.

The present study examined the expression pattern of TP73-AS1 in NSCLC and investigated the role of TP73-AS1 in the progression and drug chemoresistance of NSCLC. It also provided a potential pathway to explain the mechanism of TP73-AS1 action in NSCLC to further understand the pathogenic processes of NSCLC.

\section{Materials and methods}

Lung cancer specimens. A total of 50 lung cancer specimens and paired normal tissues within $5 \mathrm{~cm}$ of the tumor were collected from patients at The First People's Hospital of Tianmen. Patients' sample collection and 5-year overall survival investigation were conducted between January 2013 and November 2018. All specimens were promptly placed in liquid nitrogen following surgery, then stored at $-80^{\circ} \mathrm{C}$. All patients provided written informed consent prior to surgery. The present study was approved by the Ethics Committee of The First People's Hospital of Tianmen. Clinical variables were obtained from the medical records of the patients, including age, sex, tumor size, and prognosis, to determine the association between TP73-AS1 expression and clinicopathological characteristics (Table I). The demarcation of high expression or low expression of TP73-AS1 was performed according to the mean value of TP73-AS1 expression in all cases. The clinical stages of the NSCLC tissue samples were confirmed according to the tumor-node-metastasis (TNM) classification of the 7 th edition of the American Joint Committee on Cancer/Union for International Cancer Control (27).

Chromogenic in situ hybridization (CISH) assay. CISH was performed using the ZytoDot CISH Implementation kit with the commercially available digoxigenin (DIG)-labeled ZytoDot CISH probes (ZytoVision $\mathrm{GmbH}$ ). Tissue preparation (3-5 $\mu \mathrm{m}$ microtome sections), pre-treatment, hybridization and quenching were performed according to the manufacturer's instructions prior to the detection of hybridized DIG-labeled probes. NSCLC tissue microarray (TMA) manufacturing was outsourced to Outdo Biotech, Co., Ltd. The TMA consisted of 50 pairs of NSCLC tissues and matched, adjacent, normal tissues (within $5 \mathrm{~cm}$ of the tumor). Probes labeled with DIG result in permanent diaminobenzidine (DAB) brown-colored, distinct, dot-shaped signals, which are clearly distinguishable from the background counterstained with hematoxylin.

Cell lines and culture. The HCC827, H522, and H23 NSCLC cell lines, and the $16 \mathrm{HBE}$ bronchial epithelial cell were purchased from BeNa Culture Collection. HCC827, H522 and H23 were cultured in RPMI-1640 supplemented with $10 \%$ FBS (both from Gibco; Thermo Fisher Scientific, Inc.) at $37^{\circ} \mathrm{C}$ containing $5 \% \mathrm{CO}_{2}$. $16 \mathrm{HBE}$ cells were cultured in DMEM (Gibco; Thermo Fisher Scientific, Inc.) and $10 \% \mathrm{FBS}$ at $37^{\circ} \mathrm{C}$ containing $5 \% \mathrm{CO}_{2}$.

Cell transfection. Small interfering (si)RNA against TP73-AS1 (si-TP73-AS1, \#1: 5'-TAAGGTTATCCGAATAACGGT ATCGTT-3'; \#2: 5'-CCTGCTGCCTCTCCAAGAGACTGC TATTA-3' and \#3: 5'-GCAGTCGGGGCTGACGGCGG-3'), miR-34a-5p mimic [miR-34a-5p: (5'-UGGCAGUGUCUU AGCUGGUUGU-3')], miR-34a-5p inhibitor (anti-miR-34a-5p: 5'-ACAACCAGCTAAGACACUTCCA-3') and their negative controls [si-NC (5'-CCTCCACGTCACGTATAGTGACAT T-3'), miR-NC (5'-UUCUCCGAACGUGUCACGUTT-3') and anti-NC (5'-UUCUCCGAACGUGUCACGUTT-3'), respectively] were synthesized by Guangzhou RiboBio Co., Ltd. TP73-AS1 overexpression vector, TRIM29 overexpression (pcDNA-TRIM29) and pcDNA empty vector were obtained from Sangon Biotech Co., Ltd. Transient transfection with $40 \mathrm{nM}$ of the indicated oligonucleotides or $1 \mu \mathrm{g}$ plasmids was performed using Lipofectamine ${ }^{\circledR} 2000$ reagent (Invitrogen; Thermo Fisher Scientific, Inc.). Subsequent experiments were conducted $48 \mathrm{~h}$ following transfection.

Reverse transcription-quantitative PCR (RT-qPCR). Total RNA was isolated using TRIzol ${ }^{\circledR}$ reagent (Invitrogen; Thermo Fisher Scientific, Inc.) and cDNA was synthesized using the PrimeScript RT reagent kit (Takara Biotechnology Co., Ltd.) for TP73-AS1 and TRIM29, or with the One Step PrimeScript miRNA cDNA Synthesis kit (Takara Biotechnology Co., Ltd.) for miR-34a-5p. RT-qPCR was then performed using the SYBR Premix Ex Taq II kit (Takara Biotechnology Co., Ltd.) on an ABI 7900 system (Applied Biosystems; Thermo Fisher Scientific, Inc.), according to the manufacturer's instructions. Expression levels were calculated using the $2^{-\Delta \Delta \mathrm{Cq}}$ method (28) and normalized to GAPDH or small nuclear RNA U6. Primers sequences were as follows: TP73-AS1 forward, 5'-CCGGTT TTCCAGTTCTTGCA-3' and reverse, 5'-GCCTCACAG GGAAACTTCATG-3'; TRIM29 forward, 5'-TTGCATGTT CCAGGAGCACAAGAAT-3', and reverse, 5'-CAATGCACC AAATTCCTGCAGAAACA-3'; GAPDH forward, 5'-ACC ACAGTCCATGCCATCAC-3'; and reverse, 5'-TCCACCACC CTGTTGCTGTA-3'. The primers for miR-34a-5p (forward, 5'-ACACTCCAGCTGGGTGGCAGTGTCTTAGC-3'; and reverse, 5'-CTCAACTGGTGTCGTGGAGTCGGCAATTCA GTTGAGACAACCA-3') and U6 (forward, 5'-CTCGCTTCG GCAGCACA-3'; and reverse, 5'-AACGCTTCACGAATTTGC GT-3') were purchased from Guangzhou RiboBio Co., Ltd. 
Table I. Association between TP73-AS1 expression level and clinicopathological characteristics of patients with non-small cell lung cancer.

TP73-AS1 expression

\begin{tabular}{|c|c|c|c|c|}
\hline & & & & \\
\hline Characteristics & No. of patients & High, $n$ & Low, n & P-value \\
\hline Age, years & & & & 0.156 \\
\hline$<60$ & 27 & 11 & 16 & \\
\hline$\geq 60$ & 23 & 14 & 9 & \\
\hline Sex & & & & 0.774 \\
\hline Male & 29 & 15 & 14 & \\
\hline Female & 21 & 10 & 11 & \\
\hline Tumor size, $\mathrm{cm}$ & & & & $0.009^{\mathrm{a}}$ \\
\hline$<5$ & 31 & 11 & 20 & \\
\hline$\geq 5$ & 19 & 14 & 5 & \\
\hline Histology & & & & 0.569 \\
\hline Squamous & 28 & 13 & 15 & \\
\hline Adenoma & 22 & 12 & 10 & \\
\hline Differentiation & & & & 0.089 \\
\hline Well/moderately & 26 & 10 & 16 & \\
\hline Poorly & 24 & 15 & 9 & \\
\hline Lymph node metastasis & & & & $0.007^{\mathrm{a}}$ \\
\hline No & 33 & 12 & 21 & \\
\hline Yes & 17 & 13 & 4 & \\
\hline TNM stage & & & & $0.021^{\mathrm{a}}$ \\
\hline $\mathrm{I}+\mathrm{II}$ & 20 & 6 & 14 & \\
\hline III+IV & 30 & 19 & 11 & \\
\hline
\end{tabular}

${ }^{\mathrm{a}} \mathrm{P}<0.05$.

Cell proliferation and drug resistance analysis. Cell proliferation and viability was performed using a Cell Counting Kit-8 (CCK-8; Beyotime Institute of Biotechnology). Transfected cells were seeded into 96-well plates $(2,000$ cells/well) and $10 \mu \mathrm{l}$ CCK-8 solution was added into each well at different time points post-transfection $(0,24,48$ and $72 \mathrm{~h})$ for another $2 \mathrm{~h}$. The absorbance at $450 \mathrm{~nm}$ was measured using a microplate reader (Bio-Rad Laboratories, Inc.). Cells were planted into 96-well plates $(10,000$ cells/well) and then treated with varying concentrations of cisplatin (DDP) $(0,1,2,4,8,16$, 32,64 and $128 \mu \mathrm{g} / \mathrm{ml}$ ) for $48 \mathrm{~h}$, and the concentration of DDP resulting in $50 \%$ inhibition of growth $\left(\mathrm{IC}_{50}\right)$ was obtained from the dose-response curve.

Cell apoptosis analysis. Apoptosis was assessed using flow cytometry with a Cell Apoptosis kit (Invitrogen; Thermo Fisher Scientific, Inc.), according to the manufacturer's protocol. A total of $1 \times 10^{6}$ transfected HCC827 and H522 cells were washed with cold PBS and re-suspended in $1 \mathrm{X}$ Annexin binding buffer. Next, $5 \mu$ l fluorescein isothiocyanate (FITC)-conjugated Annexin V and $1 \mu 1$ propidium iodide at $100 \mu \mathrm{g} / \mathrm{ml}$ were added to $100 \mu \mathrm{l}$ cell suspension for $15 \mathrm{~min}$ at room temperature. Apoptotic cells were analyzed using the S3 ${ }^{\mathrm{TM}}$ Cell Sorter (Bio-Rad Laboratories, Inc.) and FlowJo software v7.6 (FlowJo LLC).
Cell migration and invasion analysis. Cell migration and invasion was investigated using the Transwell assay, and 24-well chambers (Corning Inc.). For migration, transfected HCC827 and H522 cells (10,000 cells/well) in serum-free RPIM medium were placed into the top of chambers without Matrigel $^{\mathrm{TM}}$ (Corning Inc.). For invasion assays, chambers were pre-coated with Matrigel ${ }^{\mathrm{TM}}$ at $4^{\circ} \mathrm{C}$ overnight, and then the transfected cells $(40,000 \mathrm{cell} / \mathrm{well})$ were seeded into the upper chambers coated with Matrigel ${ }^{\mathrm{TM}}$. In either assay, RPMI medium containing 10\% FBS was added to the bottom chambers. After a 24-h incubation, the migrated or invaded cells on the bottom surface were fixed with $90 \%$ methanol for 20 min and stained with crystal violet (Beyotime Institute of Biotechnology) for $10 \mathrm{~min}$ at room temperature. Finally, three randomly selected fields were chosen to evaluate the number of cells under a light microscope (magnification, x100; Olympus Corporation).

Bioinformatics analysis. The microRNA.org (version: 2010; http://www.microrna.org/microrna/getDownloads.do) and Starbase (version: 3.0; http://starbase.sysu.edu.cn/agoClipRNA. php? source=circRNA) online tools were used to predict the targets and binding sites between IncRNA and miRNA, or miRNA and mRNA, respectively. Then, the expression of these predicted miRNAs was detected in cells with TP73-AS1 
A

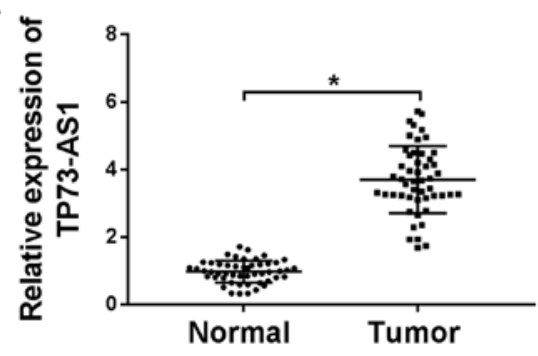

C

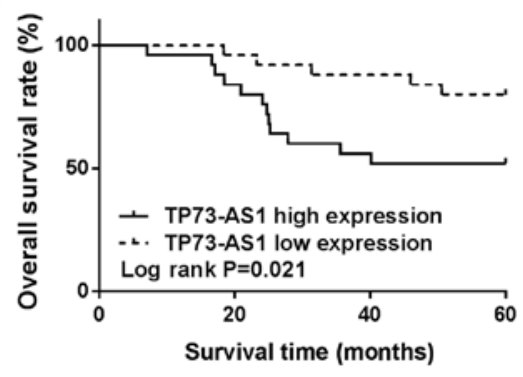

B

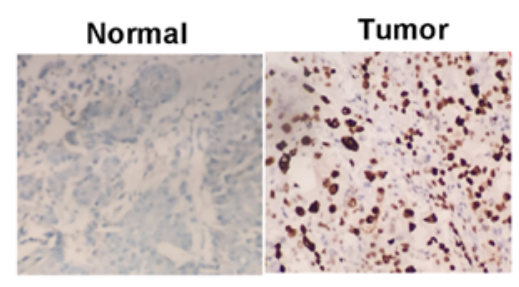

D

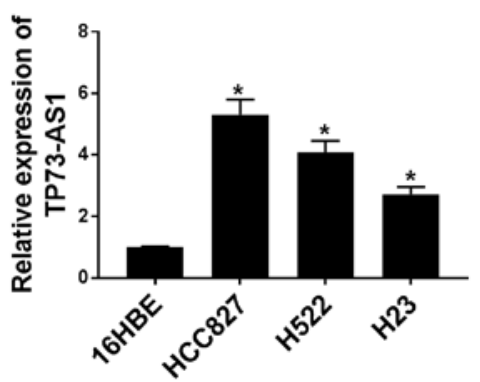

Figure 1. TP73-AS1 is upregulated in lung cancer tissues and cell lines. TP73-AS1 was expressed at higher levels in tumor tissues, compared with that in normal tissue, as determined by (A) reverse transcription-quantitative PCR and (B) chromogenic in situ hybridization (magnification, $\mathrm{x} 100$ ). (C) High expression of TP73-AS1 was associated with improved overall survival rates. (D) TP73-AS1 was upregulated in lung cancer cell lines. * $<0.05$. TP73-AS1, tumor protein 73 antisense RNA 1.

knockdown to monitor which miRNAs were upregulated. Similarly, the expression of predicted mRNAs was detected in cells with miR-34a-5p enrichment to monitor which mRNAs were downregulated, using RT-qPCR assay as detailed above.

Dual-luciferase reporter assay. The wild-type (WT) and mutant (MUT) 3'untranslated region (3'UTR) sequences of TP73-AS1 and TRIM29 containing putative binding sites with miR-34a-5p (400 bp, containing 200 bp sequences before or after binding sites) were amplified by PCR and introduced into the luciferase reporter vector PGL3 (Promega Corporation) to generate fusion plasmids, referred to as TP73-AS1-WT, TP73-AS1-MUT, TRIM29-WT and TRIM29-MUT. These fusion plasmids were transfected into HCC827 and H522 cells together with miR-34a-5p mimic or miR-NC using Lipofectamine ${ }^{\circledR} 2000$ reagent (Invitrogen; Thermo Fisher Scientific, Inc.). After a 48-h transfection, cells were collected and luciferase activity was detected using the dual $\mathrm{Glo}^{\mathrm{TM}}$ Luciferase Assay System (Promega Corporation), according to the manufacturer's protocol. Relative luciferase activity was expressed as normalization of Renilla luciferase activity to firefly luciferase activity.

Western blot analysis. Total protein was isolated using RIPA lysis buffer (Beyotime Institute of Biotechnology) and quantified using BCA Protein Assay kit (Beyotime Institute of Biotechnology). Equal amounts $(40 \mu \mathrm{g})$ of protein were electrophoresed using 12\% SDS-PAGE, then transferred onto PVDF membranes on ice (Bio-Rad Laboratories, Inc.). Next, the membranes were blocked with $5 \%$ skimmed milk for $1 \mathrm{~h}$ at room temperature and incubated with primary antibodies overnight at $4^{\circ} \mathrm{C}$. The following day, the membranes were washed with Tris-buffered saline + Tween-20 buffer, and incubated with a secondary antibody for $2 \mathrm{~h}$. The protein blots were then visualized using an enhanced chemiluminescence kit (Beyotime Institute of Biotechnology) on a ChemiDoc MP imaging system (Bio-Rad Laboratories, Inc.). All antibodies were purchased from Abcam, including primary antibodies against TRIM29 (cat. no. ab244380; 1:1,000) and GAPDH (cat. no. ab181602; 1:10,000) and the HRP-conjugated secondary antibody (cat no. ab205718; 1:5,000). The protein bands were quantified using the ImageJ software (version 1.46; National Institutes of Health).

Statistical analysis. All experiments were performed at least three times. Differences between two groups were assessed using paired and unpaired Student's t-test. ANOVA was used for multi-group comparisons, followed by Tukey's post hoc test. Overall survival was evaluated using the Kaplan-Meier method and analyzed by log-rank test. The linear association between variables was analyzed using Pearson correlation coefficient. Statistical analysis was conducted using SPSS v17.0 software (SPSS, Inc.). Data are presented as the mean \pm SD. $\mathrm{P}<0.05$ was considered to indicate a statistically significant difference.

\section{Results}

TP73-AS1 is upregulated in NSCLC tissue and cell lines. Statistical analysis of clinicopathologic features demonstrated that high TP73-AS1 expression was significantly associated with large tumor size, positive lymph node metastasis and advanced TNM stage (Table I). To determine whether the expression of TP73-AS1 was altered in NSCLC, RT-qPCR was performed in tumor samples and normal tissue $(n=50$ in each group). Compared with that in normal tissue, TP73-AS1 was expressed in tumor tissues at significantly higher levels (Fig. 1A). CISH was also used to detect the expression of 

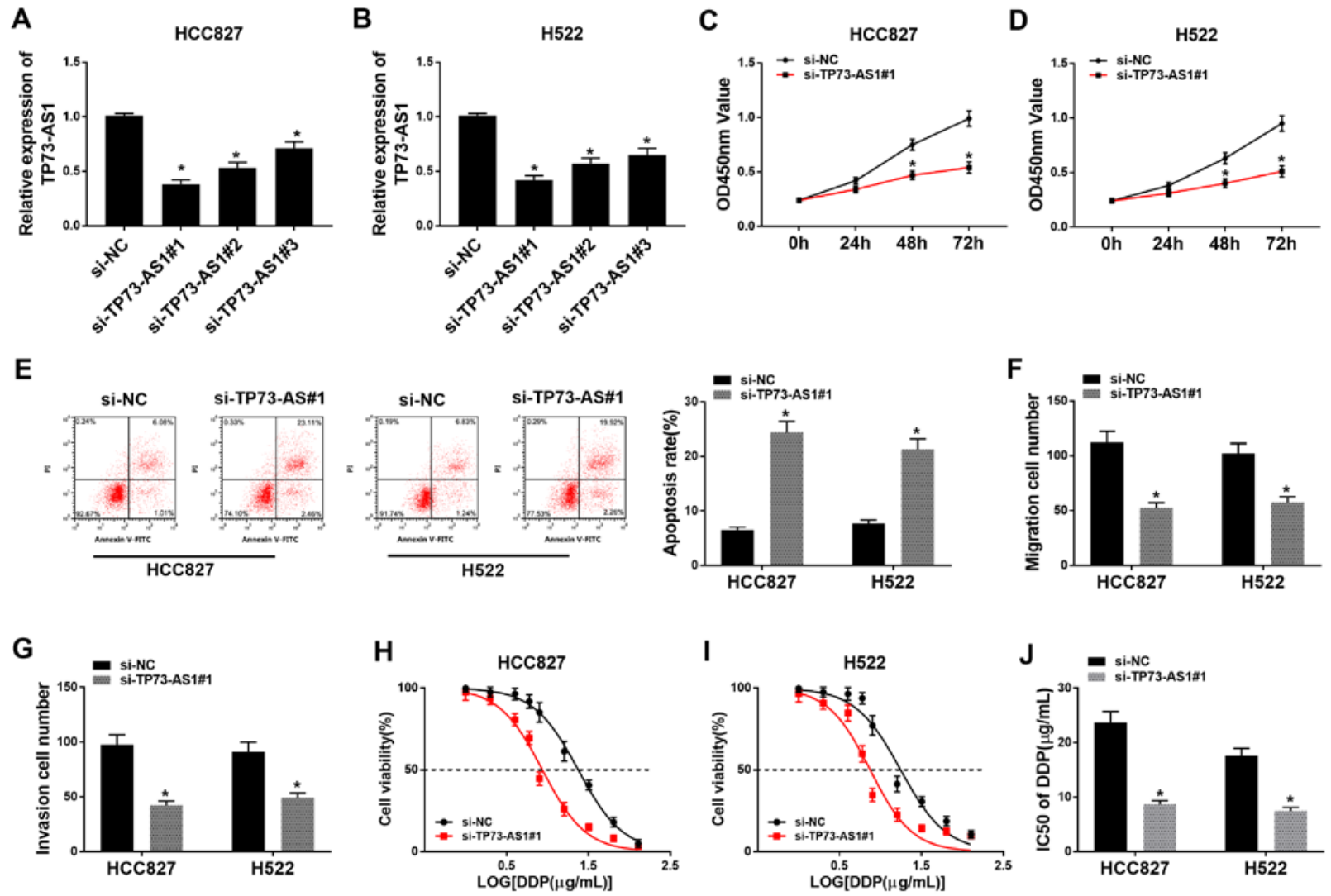

Figure 2. TP73-AS1 knockdown inhibits proliferation, migration and invasion, and promoted apoptosis and drug sensitivity. The transfection efficiency of TP73-AS1 was validated using reverse transcription-quantitative PCR in (A) HCC827 and (B) H522 cells. "P<0.05 vs. si-NC. TP73-AS1 knockdown suppressed cell proliferation in (C) HCC827 and (D) H522 cells. (E) TP73-AS1 knockdown promoted cell apoptosis. (F and G) TP73-AS1 knockdown inhibited cell migration and invasion (magnification, x100). Cell viability was detected in (H) HCC827 and (I) H522 cells following si-TP73-AS1 transfection and DDP treatment. (J) TP73-AS1 knockdown reduced the $\mathrm{IC}_{50}$ following DDP treatment in lung cancer cell lines. " $\mathrm{P}<0.05$. TP73-AS1, tumor protein 73 antisense RNA 1; DDP, cisplatin; si, small interfering; OD, optical density; NC, negative control; PI, propidium iodide; FITC, fluorescein isothiocyanate.

TP73-AS1 in NSCLC tumor tissues relative to adjacent normal tissues, which revealed a high level of TP73-AS1 in NSCLC tissues (Fig. 1B). Moreover, Kaplan-Meier curves and log-rank analysis indicated that the overall survival rate of patients with NSCLC presenting with high TP73-AS1 levels was lower, compared with patient with low TP73-AS1 expression levels (Fig. 1C).

TP73-AS1 was also highly expressed in NSCLC cell lines, including HCC827, H522, and H23, compared with that in the 16HBE bronchial epithelial cell line (Fig. 1D). In particular, expression of TP73-AS1 was significantly higher in the $\mathrm{HCC} 827$ and $\mathrm{H} 522$ cell lines relative to the $\mathrm{H} 23$ cell line. Therefore, the HCC827 and H522 cell lines were selected for subsequent experiments. Altogether, these findings indicated that increased TP73-AS1 expression levels could play a role in NSCLC progression.

TP73-AS1 knockdown inhibits proliferation, migration and invasion but induced apoptosis of NSCLC cells, and enhances sensitivity to DDP. To determine the potential role of TP73-AS1 in NSCLC cells, si-TP73-AS1 was transfected into HCC827 and $\mathrm{H} 522$ cells for functional analysis. The mRNA expression level of TP73-AS1 was reduced following transfection with si-TP73-AS1\#1, si-TP73-AS1\#2 and si-TP73-AS1\#3 transfection, and the largest decrease was in the si-TP73-AS1\#1 group, in both cell lines (Fig. 2A and B). Therefore, si-TP73-AS1\#1 was used in subsequent experiments.

Cell proliferation was significantly decreased following si-TP73-AS1 transfection (Fig. 2C and D). By contrast, apoptosis was significantly increased in HCC827 and H522 cells transfected with si-TP73-AS1 (Fig. 2E). In addition, the numbers of migrating and invading cells were significantly reduced in the NSCLC cell lines following transfection with si-TP73-AS1 (Fig. 2F-G). In addition, the viability of HCC827 and H525 cells transfected with si-TP73-AS1 was reduced with increasing DDP concentrations, compared with that in the si-NC-transfected cells. (Fig. 2H and I). Furthermore, TP73-AS1 knockdown significantly reduced $\mathrm{IC}_{50}$ of DPP in NSCLC cells (Fig. 2J). Taken together, these results demonstrated that TP73-AS1 knockdown inhibited the growth of NSCLC cells, and increased their sensitivity to DDP.

TP73-AS1 targets miR-34a-5p and negatively regulates the expression of miR-34a-5p in NSCLC cells. To explore the mechanism underlying the effect of TP73-AS1 on NSCLC cell lines, the target miRNAs of TP73-AS1 were predicted using microRNA.org and were subsequently screened according to their expression levels. The sectional predicted target miRNAs of TP73-AS1 are shown in Table SI. A significant increase in 
A
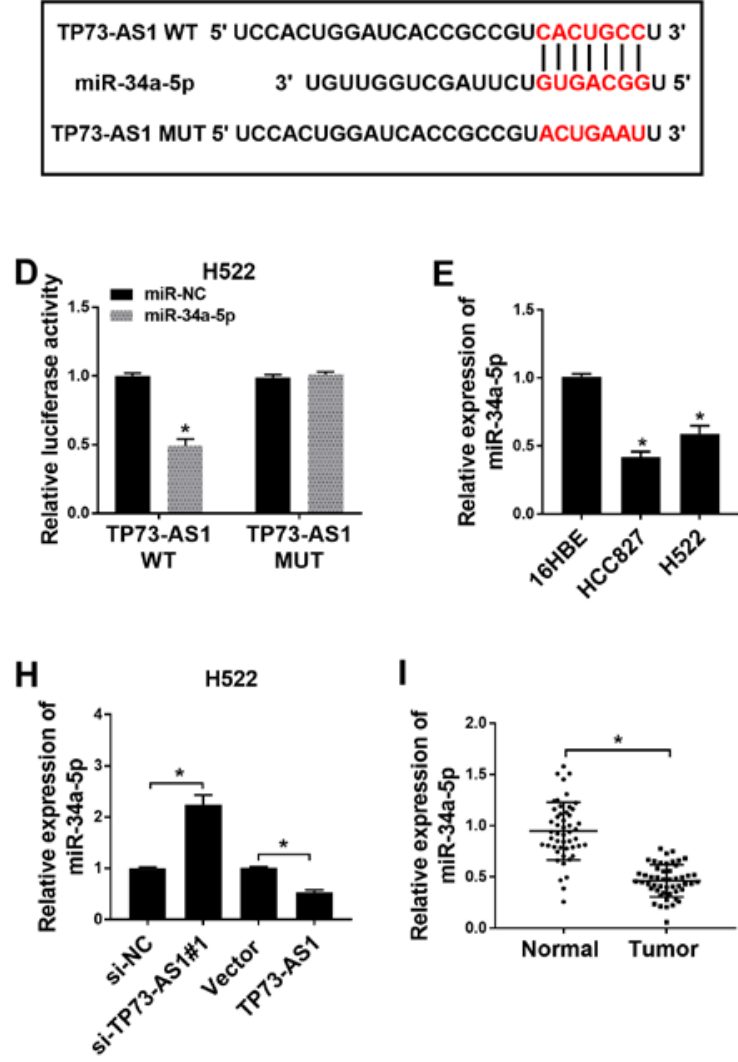

I

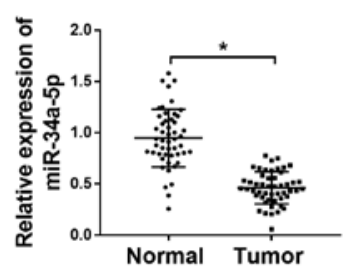

B

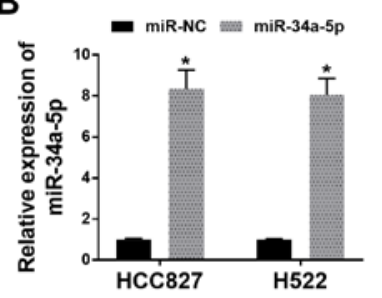

$\mathbf{F}$

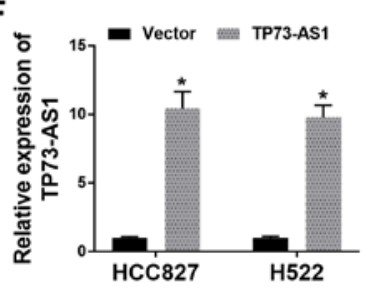

C

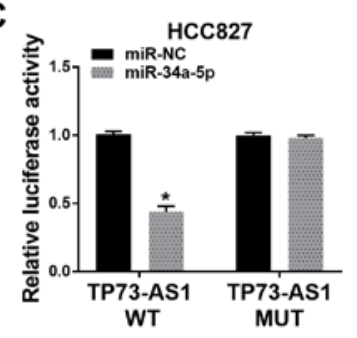

G
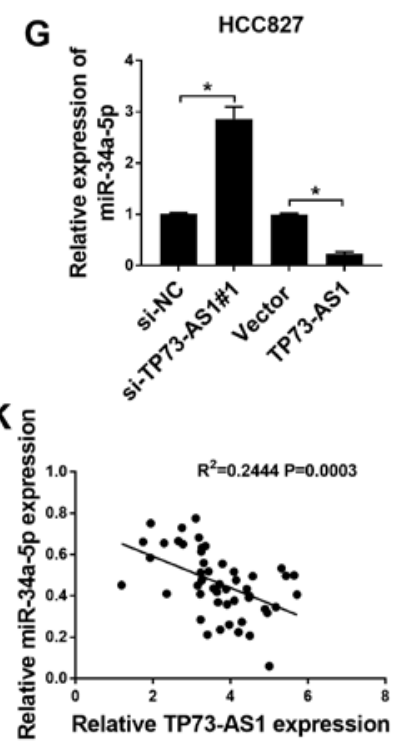

J

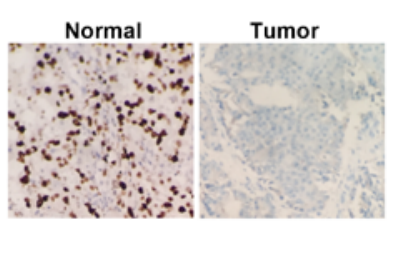

Figure 3. TP73-AS1 targets miR-34a-5p and regulates miR-34a-5p expression. (A) The binding sites between miR-34a-5p and TP73-AS1 were predicted using the microRNA.org tool. (B) Expression levels of miR-34a-5p levels following miR-34a-5p mimic transfection were determined using RT-qPCR. Dual luciferase reporter assay verified the interaction between miR-34a-5p and TP73-AS1 in (C) HCC827 and (D) H522 cells. (E) Expression of miR-34a-5p was decreased in HCC 827 and $\mathrm{H} 522$ cell lines. "P $<0.05$, vs. 16HBE (F) Efficiency of TP73-AS1 overexpression was determined using RT-qPCR. The expression level of miR-34a-5p was altered by TP73-AS1 overexpression or knockdown in (G) HCC827) and (H) H522 cells. miR-34a-5p was downregulated in non-small cell lung cancer tumor tissues compared with that in normal tissues using (I) RT-qPCR and (J) chromogenic in situ hybridization (magnification, x100), (K) miR-34a-5p expression was negatively associated with TP73-AS1 expression in NSCLC tissues. "P<0.05. TP73-AS1, tumor protein 73 antisense RNA 1; RT-qPCR, reverse transcription-quantitative PCR; miR, microRNA; NC, negative control; WT, wild-type; mut, mutant.

miR-34a-3p expression was observed following TP73-AS1 knockdown compared with that in cells transfected with si-NC, while there was no significant difference in the other predicted target miRNAs (Fig. S1). The binding sites between TP73-AS1 and miR-34a-5p were identified using microRNA. org (Fig. 3A). The expression of miR-34a-5p was subsequently found to be increased in $\mathrm{HCC} 827$ and $\mathrm{H} 522$ cells transfected with miR-34a-5p mimics, compared with that in cells transfected with miR-NC (Fig. 3B). In a dual-luciferase reporter assay, luciferase activity decreased in HCC 827 and $\mathrm{H} 522$ cells co-transfected with TP73-AS1-WT and miR-34a-5p. However, there was no difference in luciferase activity in cells co-transfected with TP73-AS1-MUT and miR-34a-5p (Fig. 3C and D).

The expression of miR-34a-5p in NSCLC cells was measured, and was found to be significantly downregulated in HCC827 and H522 cells, compared with that in 16HBE cells (Fig. 3E). The efficiency of TP73-AS1 overexpression was subsequently verified. The expression of TP73-AS1 was significantly increased in HCC827 and H522 cells transfected with TP73-AS1, compared with that in cells transfected with the empty vector (Fig. 3F). Further analysis suggested that TP73-AS1 knockdown in HCC827 and H522 cells increased miR-34a-5p expression, while TP73-AS1 overexpression reduced miR-34a-5p expression (Fig. 3G and H). Furthermore, the expression of miR-34a-5p was significantly lower in NSCLC tumor tissues compared with that in normal tissues (Fig. 3I), which was also confirmed using CISH (Fig. 3J). In addition, Pearson correlation coefficient analysis demonstrated that miR-34a-5p expression was negatively associated with TP73-AS1 expression in NSCLC tissues (Fig. 3K). These results demonstrated that miR-34a-5p was targeted by TP73-AS1.

miR-34a-5p inhibition reverses the effects of TP73-AS1 knockdown on proliferation, apoptosis, migration, invasion and drug resistance in NSCLC cells. To determine whether TP73-AS1 exerted its effects by targeting miR-34a-5p, the HCC 827 and H522 cell lines were transfected with si-TP73-AS1 and anti-miR-34a-5p. Transfection with anti-miR-34a-5p significantly reduced the expression of miR-34a-5p in HCC 827 and H522 cells, compared with that in cells transfected with anti-NC (Fig. 4A). In a CCK-8 assay, cell proliferation was inhibited 
A
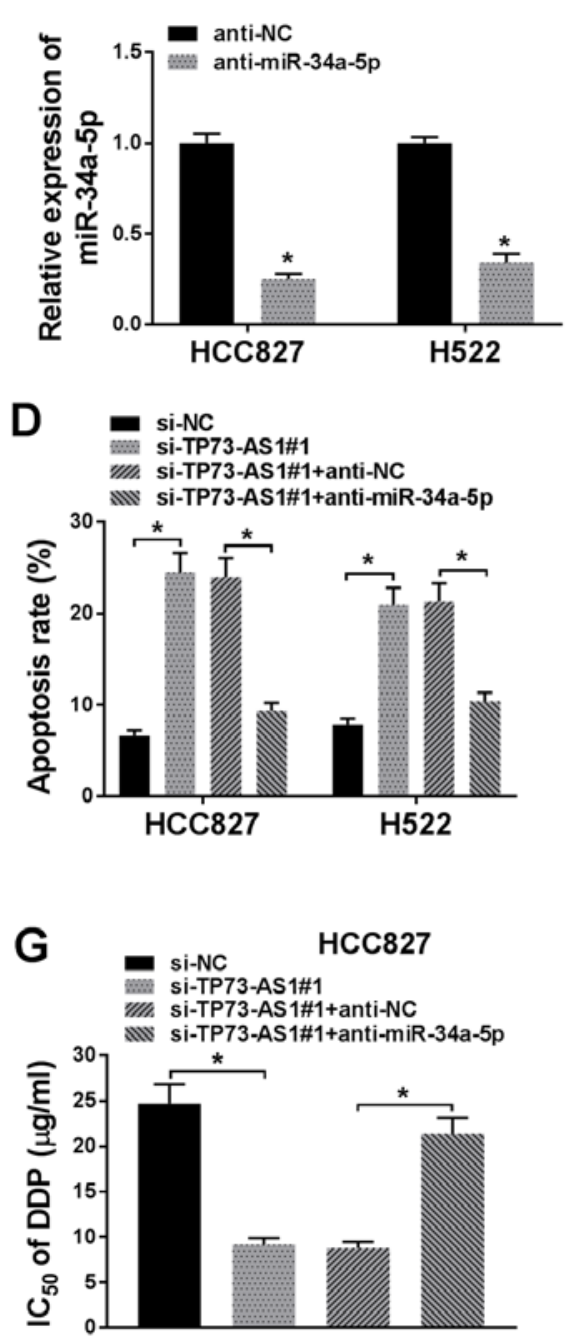

B
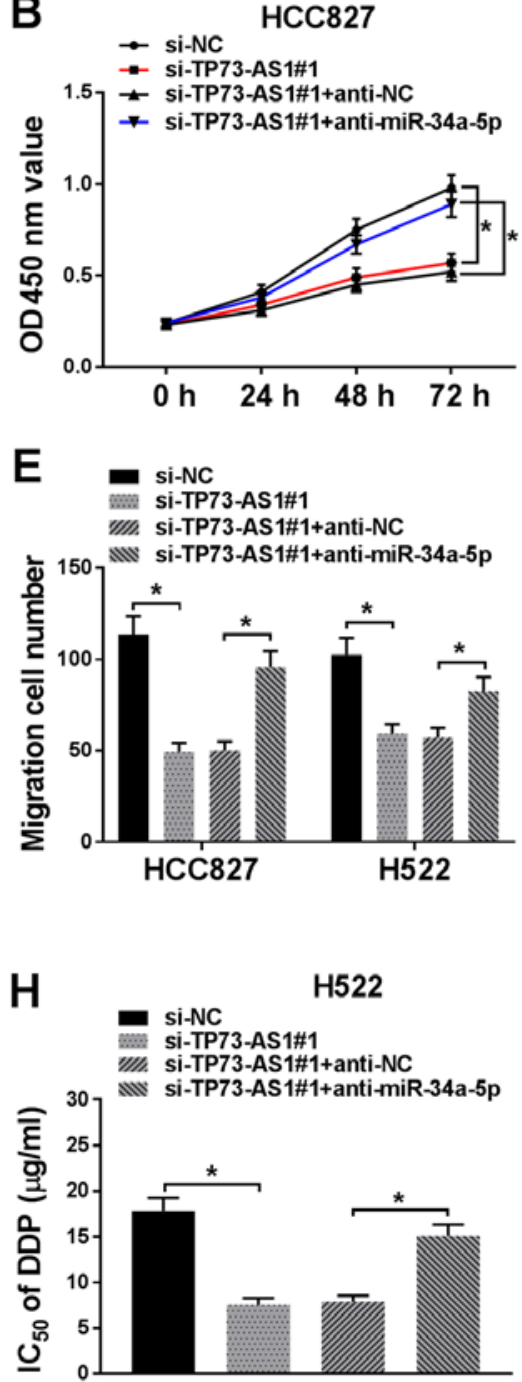

C

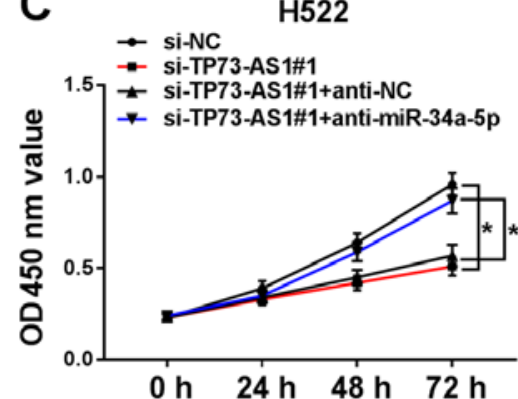

F

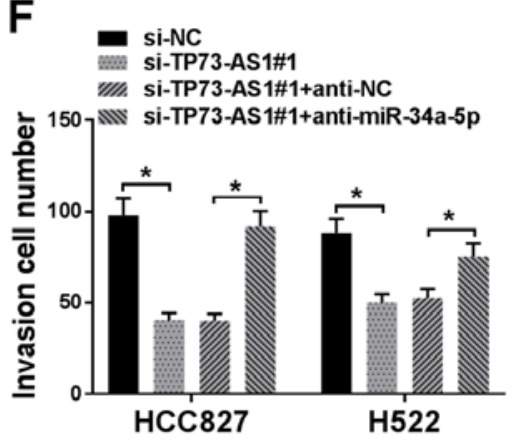

Figure 4. Inhibition of miR-34a-5p reverses the effect of TP73-AS1 knockdown in NSCLC cells. (A) Transfection of anti-miR-34a-5p decreased miR-34a-5p expression in HCC827 and H522 cells. Transfection with anti-miR-34a-5p transfection reverses the effect of si-TP73-AS1 on (B and C) proliferation, (D) apoptosis, (E) migration, (F) invasion and (G and H) DDP resistance in NSCLC cells. ${ }^{*} \mathrm{P}<0.05$. TP73-AS1, tumor protein 73 antisense RNA 1 ; NSCLC, non-small cell lung cancer; miR, microRNA; si, small interfering; DDP, cisplatin; OD, optical density; NC, negative control.

following si-TP73-AS1 transfection, compared with that in cells transfected with si-NC. However, this effect was revered following transfection with si-TP73-AS1+anti-miR-34a-5p, in both HCC827 and H522 cells (Fig. 4B and C). Apoptosis was significantly increased by TP73-AS1 knockdown but was significantly decreased by anti-miR-34a-5p (Fig. 4D). The number of migrating and invading cells was inhibited by TP73-AS1 knockdown, but rescued by miR-34a-5p inhibition (Fig. 4E and F). Furthermore, the $\mathrm{IC}_{50}$ of DDP was reduced following TP73-AS1 knockdown. By contrast, anti-miR-34a-5p increased the $\mathrm{IC}_{50}$ value in NSCLC cells (Fig. 4G and H). Taken together, these findings suggest that TP73-AS1 knockdown blocked proliferation, migration and invasion, accelerated apoptosis, and reduced resistance to DDP, by upregulating miR-34a-5p in NSCLC cells.

TRIM29 is a miR-34a-5p target in NSCLC cells. To determine whether miR-34a-5p functioned by regulating downstream genes, potential target mRNAs were obtained using Starbase. The sectional predicted target mRNAs of miR-34a-5p are shown in Table SII. A significant decrease was found in
TRIM29 expression in cells following miR-34a-5p transfection compared with that in cells transfected with miR-NC (Fig. S2). A binding site was predicted between miR-34a-5p and TRIM29 3'UTR (Fig. 5A). In a dual-luciferase reporter assay, luciferase activity was significantly reduced in HCC827 and H522 cells co-transfected with TRIM29-WT and miR-34a-5p. By contrast, TRIM29-MUT and miR-34a-5p transfection did not affect luciferase activity compared with that in the control group (Fig. 5B and C). The expression of TRIM29 at the protein level was suppressed following transfection with miR-34a-5p mimic, but enhanced by anti-miR-34a-5p transfection, in both HCC 827 and H522 cells (Fig. 5D and E). These findings suggested that TRIM29 was a target for miR-34a-5p.

TP73-AS1 knockdown inhibits TRIM29 expression by increasing the expression of $m i R-34 a-5 p$ in NSCLC cells. To determine whether TRIM29 expression was modulated by TP73-AS1 and miR-34a-5p, the expression levels of TRIM29 in HCC827 and H522 cells were investigated under 
A

TRIM29 WT 3'UTR 5' AAAGGUGCCUUACACACUGCCC 3'
miR-34a-5p 3' UGUUGGUCGAUUCUGUGACGGU 5'
TRIM29 MUT 3'UTR 5' AAAGGUGCCUUACGACUGAUUC 3'

D

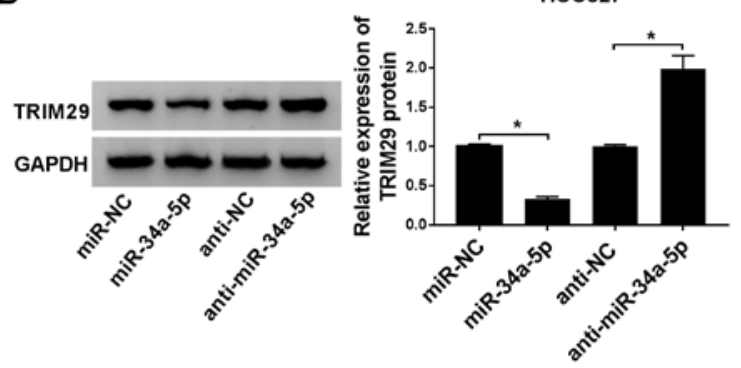

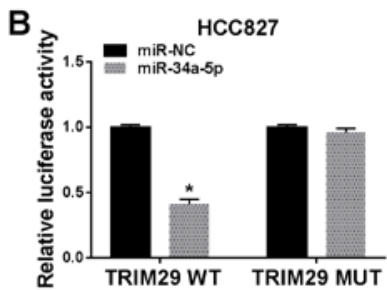

E

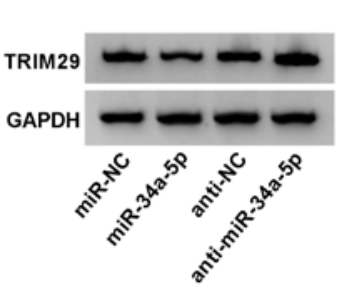

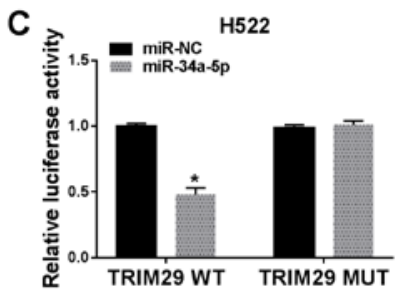

H522

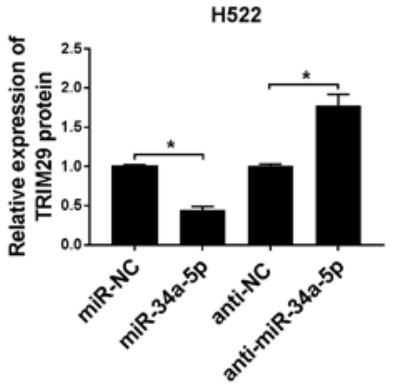

Figure 5. TRIM29 is targeted by miR-34a-5p. (A) The binding sites between miR-34a-5p and TRIM29 3'UTR were predicted by Starbase. Association between miR-34a-5p and TRIM29 was confirmed using dual-luciferase activity assay in (B) HCC827 and (C) H522 cells. Western blot analysis indicated that the protein expression level of TRIM29 was inhibited by miR-34a-5p overexpression but increased following transfection with anti-miR-34a-5p in (D) HCC827 and (E) H522 cells. ${ }^{*}$ P<0.05. TRIM29, tripartite motif-containing 29; miR, microRNA; UTR, untranslated region; WT, wild-type; mut, mutant; $\mathrm{NC}$, negative control.

A

\section{B}

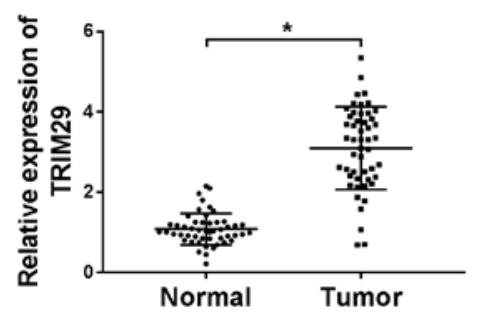

HCC827

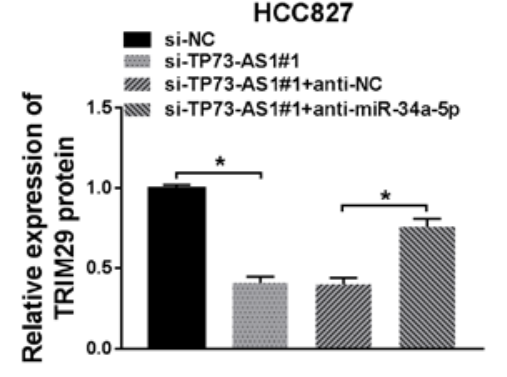

C

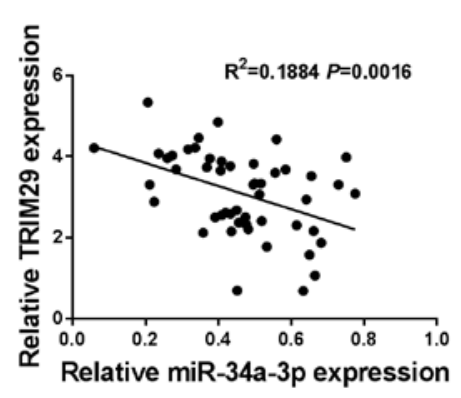

H522

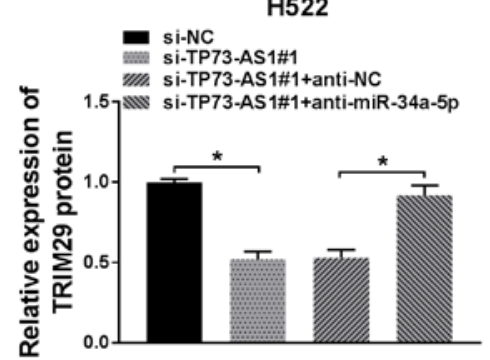

D

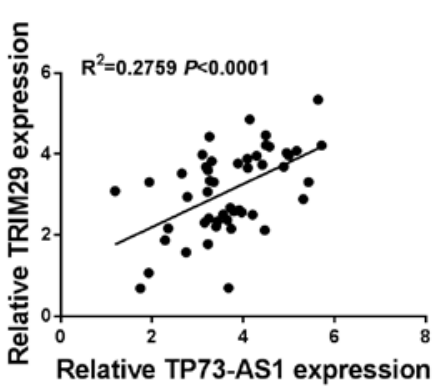

Figure 6. TP73-AS1 regulates the expression of TRIM29 via modulating miR-34a-5p in NSCLC cells. (A) TRIM29 protein expression was decreased following si-TP73-AS1 transfection but was reversed following si-TP73-AS1+anti-miR-34a-5p co-transfection. (B) TRIM29 was upregulated in NSCLC tumor tissues vs. normal tissues. TRIM29 expression was (C) negatively associated with miR-34a-5p expression and (D) positively associated with TP73-AS1 expression. "P<0.05. TP73-AS1, tumor protein 73 antisense RNA 1; TRIM29, tripartite motif-containing 29; NSCLC, non-small cell lung cancer; si, small interfering; miR, microRNA; NC, negative control.

different transfection conditions. The protein expression level of TRIM29 decreased following si-TP73-AS1 transfection. However, TRIM29 expression was restored following si-TP73-AS1+anti-miR-34a-5p co-transfection (Fig. 6A). In addition, the expression of TRIM29 was significantly higher in NSCLC tumor tissues compared with that in normal tissues (Fig. 6B). Pearson's correlation coefficient analysis demonstrated that TRIM29 expression was negatively associated with miR-34a-5p expression (Fig. 6C), but positively associated with TP73-AS1 expression (Fig. 6D). These results suggested 
A

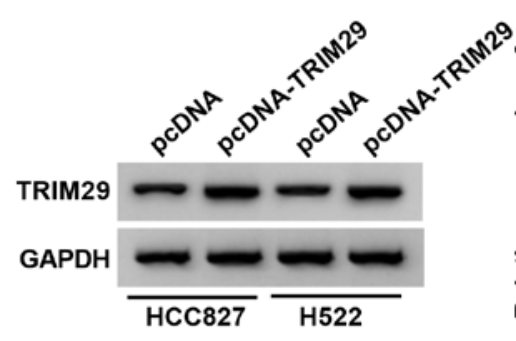

C

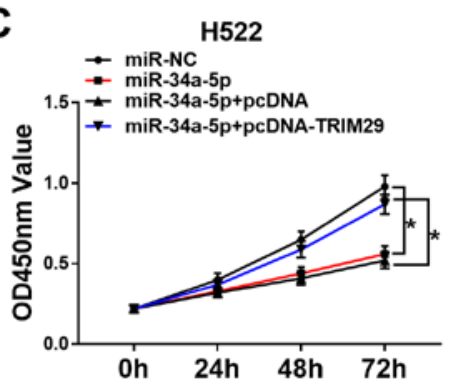

$\mathbf{F}$

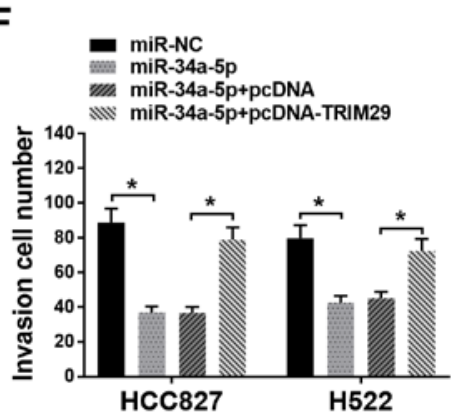

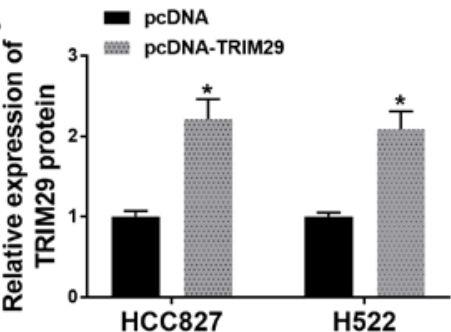

D

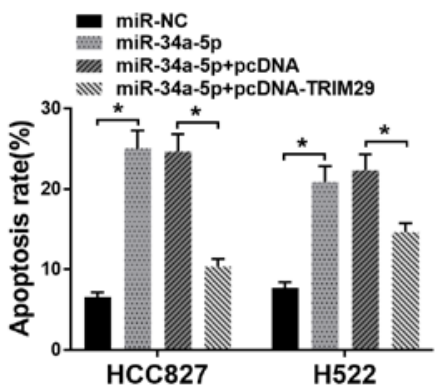

G

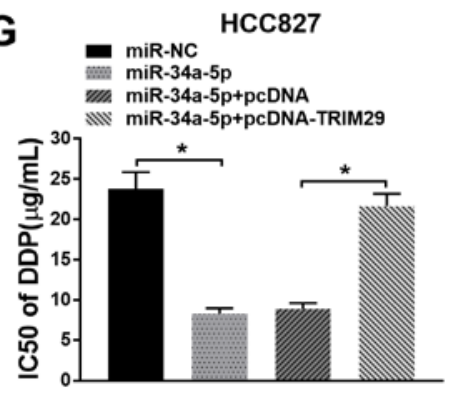

B

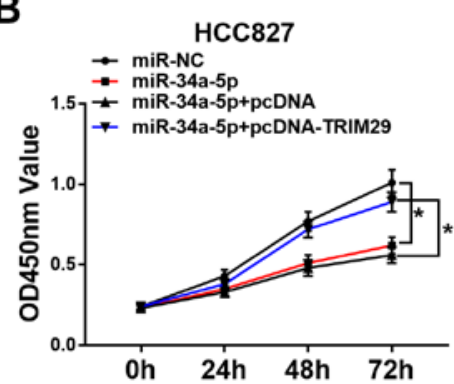

E

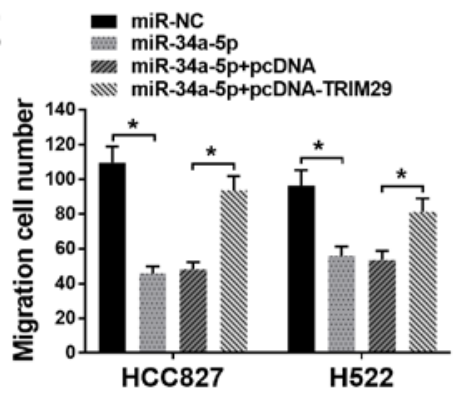

H

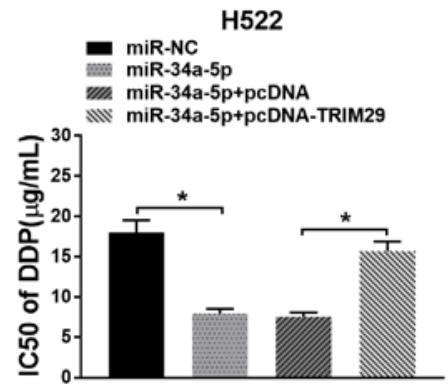

Figure 7. Overexpression of TRIM29 reverses the effects of miR-34a-5p mimics on proliferation, apoptosis, migration, invasion and DPP resistance in NSCLC cells. (A) Transfection with pcDNA-TRIM29 increased TRIM29 expression in HCC827 and H522 cell, as indicated by western blot analysis. CCK-8 assay found that miR-34a-5p+pcDNA-TRIM29 co-transfection restored cell proliferation vs. miR-34a-5p transfection alone in (B) HCC827 and (C) H522 cells. (D) TRIM29 overexpression inhibited apoptosis in NSCLC cells. Cell (E) migration and (F) invasion was suppressed following miR-34a-5p overexpression, but recovered after TRIM29 overexpression. DDP IC50 was reduced following miR-34a-5p transfection, but increased after miR-34a-5p + pcDNA-TRIM29 transfection in (G) HCC827 and (H) H522 cells. "P<0.05. TRIM29, tripartite motif-containing 29; NSCLC, non-small cell lung cancer; miR, microRNA; NC, negative control; OD, optical density.

that the expression of TRIM29 was regulated by TP73-AS1 through miR-34a-5p.

TRIM29 overexpression reverses the effects of miR-34a-5p enrichment on proliferation, apoptosis, migration, invasion and drug resistance in NSCLC cells. To further investigate the mechanism underlying the effects of TP73-AS1 on NSCLC cells, miR-34a-5p, miR-NC, miR-34a-5p+pcDNA-TRIM29 or miR-34a-5p+pcDNA were introduced into HCC827 and H522 cells. Transfection with pcDNA-TRIM29 led to a significant increase in TRIM29 protein expression compared with that in cells transfected with pcDNA (Fig. 7A). A CCK-8 assay demonstrated that NSCLC cell proliferation was reduced by miR-34a-5p mimic, but promoted following miR-34a-5p+pcDNA-TRIM29 transfection (Fig. 7B and C). Apoptosis was significantly increased following miR-34a-5p mimic transfection. However, the rates of apoptosis were reduced following miR-34a-5p+pcDNA-TRIM29 co-transfection, in both HCC 827 and H522 cells (Fig. 7D). The numbers of migrating and invading cells were significantly reduced by miR-34a-5p mimic transfection and significantly increased by TRIM29 overexpression (Fig. 7E and F). Furthermore, the $\mathrm{IC}_{50}$ of DDP was significantly decreased with miR-34a-5p mimic. By contrast, $\mathrm{IC}_{50}$ values were significantly increased by TRIM29 overexpression (Fig. 7G and H). Taken together, this indicated that miR-34a-5p overexpression inhibited NSCLC cell growth and drug resistance by downregulating TRIM29.

\section{Discussion}

Substantial progress has been made into the management and treatment of lung cancer; however, it remains the leading cause of cancer-related mortality worldwide (29). The identification of additional biomarkers are still required for the treatment of NSCLC. In the present study, TP73-AS1 was highly expressed in NSCLC cells. Functional analysis demonstrated 
that TP73-AS1 downregulation inhibited NSCLC cell growth and resistance to DDP. An interaction between miR-34a-5p, TP73-AS1 and TRIM29 was also identified. Thus, TP73-AS1 promoted tumor cell growth, invasion and drug resistance to DDP through the modulation of TRIM29 and miR-34a-5p in NSCLC cells.

Numerous molecules and genes that are abnormally expressed in tumor tissues significantly affect the occurrence and development of tumors. Previous studies have found that TP73-AS1 was associated with the development of NSCLC. For example, Zhu et al (30) found that TP73-AS1 was expressed at high levels in NSCLC tumor tissues and was associated with poor survival. In addition, increased TP73-AS1 endogenous levels promoted migration and invasion of NSCLC cells (30). Zhang et al (31) found that inhibition of TP73-AS1 reduced NSCLC cell proliferation, inhibited cell cycle progression in vitro, and blocked tumor growth in vivo. Furthermore, a previous study demonstrated that TP73-AS1 promoted temozolomide resistance in glioblastoma multiform cancer stem cells (32). However, whether TP73-ASa could affect DDP resistance in NSCLC remains unclear. In the present study, TP73-AS1 was also upregulated in NSCLC tissues and cells. Moreover, TP73-AS1 knockdown suppressed cell growth, migration and invasion, and inhibited drug resistance to DDP. These findings demonstrated that TP73-AS1 functioned as a tumor promoter in NSCLC. Reactive oxygen species (ROS) are a vital biological product of regular cell metabolism (33). ROS play an important role in numerous biological processes, such as cell survival, differentiation and apoptosis (33). Notably, apoptosis induced by intracellular production of ROS has been recognized as one of the basic antitumor mechanisms of DDP $(34,35)$. Therefore, further investigations should focus on the association between ROS production and TP73-AS1 dysregulation in NSCLC.

miR-34a-5p was identified as a target of TP73-AS1 in the present study. A previous study demonstrated that miR-34a-5p was involved in the inhibition of lung cancer progression and metastasis induced by curcumin (36). Another study, based on miRNA microarray and quantitative PCR, found that miR-34a-5p was upregulated in NSCLC tumor tissues treated with luteolin, resulting in the inhibition of tumorigenesis (37). Moreover, miR-34a was downregulated in lung cancer cells, which enhanced their sensitivity to DDP (38). miR-34a overexpression also enhances DDP sensitivity in gastric cancer (39). In agreement with these previous studies, miR-34a-5p was downregulated in NSCLC cells in the present study. In addition, miR-34a-5p overexpression reduced proliferation, migration, invasion and drug resistance to DDP, whilst inducing apoptosis in NSCLC cells. These results provide insight into the role of miR-34a-5p in the growth and DDP resistance of NSCLC cells.

Further experiments suggested that TRIM29 was targeted by miR-34a-5p. Previous studies have documented that TRIM29 functions as a tumor suppressor in several types of cancer, including breast cancer and hepatocellular carcinoma $(23,40)$. By contrast, TRIM29 appears to function as a tumor promoter in lung-related cancers, and accelerates the progression of lung cancer (41-43). Moreover, Liu et al (43) also found that TRIM29 downregulation could augment DDP chemosensitivity in lung cancer cells. Similarly, TRIM29 was upregulated in of NSCLC tissues and cell lines in the present study. TRIM29 overexpression reversed the inhibitory effect of miR-34a-5p on progression and DDP resistance in NSCLC. In conclusion, TRIM29 enhanced the growth and DDP resistance to DDP of NSCLC cells.

In summary, the expression of TP73-AS1 was increased in NSCLC tissues and cells, leading to poor overall survival. TP73-AS1 promoted NSCLC progression and resistance to DDP, which was accomplished partially by modulating the TP73-AS1/miR-34a-5p/TRIM29 axis. Thus, the present study suggested that TP73-AS1 might represent a potential biomarker in the treatment of NSCLC.

\section{Acknowledgements}

Not applicable.

\section{Funding}

No funding was received.

\section{Availability of data and materials}

The datasets used and/or analyzed during the current study are available from the corresponding author on reasonable request.

\section{Authors' contributions}

MS and HC designed the study and performed the experiments. WL and CC collated and analyzed the data. SL and HC were involved in performing the experiments. All authors read and approved the final manuscript.

\section{Ethics approval and consent to participate}

The present study was approved by the Ethical Review Committee of The First People's Hospital of Tianmen. All patients provided written informed consent prior to the start of the study.

\section{Patient consent for publication}

Not applicable.

\section{Competing interests}

The authors declare that they have no competing interests.

\section{References}

1. Siegel RL, Miller KD and Jemal A: Cancer statistics, 2019. CA Cancer J Clin 69: 7-34, 2019

2. Papaetis GS, Roussos C and Syrigos KN: Targeted therapies for non-small cell lung cancer. Curr Pharm Des 13: 2810-2831, 2007.

3. Fan WD, Zhang XQ, Guo HL, Zeng WW, Zhang N, Wan QQ, $\mathrm{Xie} \mathrm{WY}, \mathrm{Cao} \mathrm{J}$ and $\mathrm{Xu} \mathrm{CH}$ : Bioinformatics analysis reveals connection of squamous cell carcinoma and adenocarcinoma of the lung. Asian Pac J Cancer Prev 13: 1477-1482, 2012.

4. Younes RN, Pereira JR, Fares AL and Gross JL: Chemotherapy beyond first-line in stage IV metastatic non-small cell lung cancer. Rev Assoc Med Bras (1992) 57: 686-691, 2011.

5. Gainor JF and Shaw AT: Novel targets in non-small cell lung cancer: ROS1 and RET fusions. Oncologist 18: 865-875, 2013. 
6. Spizzo R, Almeida MI, Colombatti A and Calin GA: Long non-coding RNAs and cancer: A new frontier of translational research? Oncogene 31: 4577-4587, 2012.

7. Dimartino D, Colantoni A, Ballarino M, Martone J, Mariani D, Danner J, Bruckmann A, Meister G, Morlando M and Bozzoni I: The long non-coding RNA lnc-31 interacts with Rock1 mRNA and mediates its YB-1-dependent translation. Cell Rep 23 733-740, 2018.

8. Shi X, Liu Z, Liu Z, Feng X, Hua F, Hu X, Wang B, Lu K and Nie F: Long noncoding RNA PCAT6 functions as an oncogene by binding to EZH2 and suppressing LATS2 in non-small-cell lung cancer. EBioMedicine 37: 177-187, 2018.

9. Lu Q, Shan S, Li Y, Zhu D, Jin W and Ren T: Long noncoding RNA SNHG1 promotes non-small cell lung cancer progression by up-regulating MTDH via sponging miR-145-5p. FASEB J 32: 3957-3967, 2018

10. Tan J and Yang L: Long noncoding RNA VPS9D1-AS1 overexpression predicts a poor prognosis in non-small cell lung cancer Biomed Pharmacother 106: 1600-1606, 2018.

11. Varon M, Levy T, Mazor G, Ben David H, Marciano R, Krelin Y, Prasad M, Elkabets M, Pauck D, Ahmadov U, et al: The long noncoding RNA TP73-AS1 promotes tumorigenicity of medulloblastoma cells. Int J Cancer 145: 3402-3413, 2019.

12. Liu G, Zhao X, Zhou J, Cheng X, Ye Z and Ji Z: lncRNA TP73-AS1 promotes cell proliferation and inhibits cell apoptosis in clear cell renal cell carcinoma through repressing KISS1 expression and inactivation of PI3K/Akt/mTOR signaling pathway. Cell Physiol Biochem 48: 371-384, 2018.

13. Bartel DP: MicroRNAs: Target recognition and regulatory functions. Cell 136: 215-233, 2009.

14. Bartel DP: MicroRNAs: Genomics, biogenesis, mechanism, and function. Cell 116: 281-297, 2004.

15. Shukla GC, Singh J and Barik S: MicroRNAs: Processing, maturation, target recognition and regulatory functions. Mol Cell Pharmacol 3: 83-92, 2011.

16. Macfarlane LA and Murphy PR: MicroRNA: Biogenesis, function and role in cancer. Curr Genomics 11: 537-561, 2010.

17. Gao J, Li N, Dong Y, Li S, Xu L, Li X, Li Y, Li Z, Ng SS, Sung JJ, et al: $\mathrm{miR}-34 \mathrm{a}-5 \mathrm{p}$ suppresses colorectal cancer metastasis and predicts recurrence in patients with stage II/III colorectal cancer. Oncogene 34: 4142-4152, 2015.

18. Zhong Z, Zhou F, Wang D, Wu M, Zhou W, Zou Y, Li J, Wu L and Yin X: Expression of KLF9 in pancreatic cancer and its effects on the invasion, migration, apoptosis, cell cycle distribution, and proliferation of pancreatic cancer cell lines. Oncol Rep 40 3852-3860, 2018.

19. Sun Z, Zhang B and Cui T: Long non-coding RNA XIST exerts oncogenic functions in pancreatic cancer via miR-34a-5p. Oncol Rep 39: 1591-1600, 2018

20. Kosaka Y, Inoue H, Ohmachi T, Yokoe T, Matsumoto T, Mimori K, Tanaka F, Watanabe M and Mori M: Tripartite motif-containing 29 (TRIM29) is a novel marker for lymph node metastasis in gastric cancer. Ann Surg Oncol 14: 2543-2549, 2007.

21. Masuda Y, Takahashi H, Sato S, Tomomori-Sato C, Saraf A Washburn MP, Florens L, Conaway RC, Conaway JW and Hatakeyama S: TRIM29 regulates the assembly of DNA repair proteins into damaged chromatin. Nat Commun 6: 7299, 2015.

22. Napolitano LM and Meroni G: TRIM family: Pleiotropy and diversification through homomultimer and heteromultimer formation. IUBMB Life 64: 64-71, 2012.

23. Ai L, Kim WJ, Alpay M, Tang M, Pardo CE, Hatakeyama S, May WS, Kladde MP, Heldermon CD, Siegel EM and Brown KD: TRIM29 suppresses TWIST1 and invasive breast cancer behavior. Cancer Res 74: 4875-4887, 2014.

24. Qiu F, Xiong JP, Deng J and Xiang XJ: TRIM29 functions as an oncogene in gastric cancer and is regulated by miR-185. Int J Clin Exp Pathol 8: 5053-5061, 2015.

25. Xu J, Li Z, Su Q, Zhao J and Ma J: TRIM29 promotes progression of thyroid carcinoma via activating P13K/AKT signaling pathway. Oncol Rep 37: 1555-1564, 2017.

26. Tan ST, Liu SY and Wu B: TRIM29 overexpression promotes proliferation and survival of bladder cancer cells through NF- $\kappa B$ signaling. Cancer Res Treat 48: 1302-1312, 2016.
27. Edge SB and Compton CC: The American joint committee on cancer: The 7th edition of the AJCC cancer staging manual and the future of TNM. Ann Surg Oncol 17: 1471-1474, 2010.

28. Livak KJ and Schmittgen TD: Analysis of relative gene expression data using real-time quantitative PCR and the 2(-Delta Delta $\mathrm{C}(\mathrm{T})$ ) method. Methods 25: 402-408, 2001

29. Balata H, Fong KM, Hendriks LE, Lam S, Ostroff JS, Peled N, $\mathrm{Wu} \mathrm{N}$ and Aggarwal C: Prevention and early detection for NSCLC: Advances in thoracic oncology 2018. J Thorac Oncol 14: 1513-1527, 2019.

30. Zhu D, Zhou J, Liu Y, Du L, Zheng Z and Qian X: lncRNA TP73-AS1 is upregulated in non-small cell lung cancer and predicts poor survival. Gene 710: 98-102, 2019.

31. Zhang L, Fang F and He X: Long noncoding RNA TP73-AS1 promotes non-small cell lung cancer progression by competitively sponging miR-449a/EZH2. Biomed Pharmacother 104: 705-711, 2018.

32. Mazor G, Levin L, Picard D, Ahmadov U, Carén H, Borkhardt A, Reifenberger G, Leprivier G, Remke M and Rotblat B: The lncRNA TP73-AS1 is linked to aggressiveness in glioblastoma and promotes temozolomide resistance in glioblastoma cancer stem cells. Cell Death Dis 10: 246, 2019.

33. Chen B, Shen Z, Wu D, Xie X, Xu X, Lv L, Dai H, Chen J and Gan X: Glutathione peroxidase 1 promotes NSCLC resistance to cisplatin via ROS-induced activation of PI3K/AKT pathway. Biomed Res Int 2019: 7640547, 2019.

34. Zhang Y, Zheng S, Zheng JS, Wong KH, Huang Z, Ngai SM, Zheng W, Wong YS and Chen T: Synergistic induction of apoptosis by methylseleninic acid and cisplatin, the role of ROS-ERK/AKT-p53 pathway. Mol Pharm 11: 1282-1293, 2014.

35. Tonino SH, van Laar J, van Oers MH, Wang JY, Eldering E and Kater AP: ROS-mediated upregulation of Noxa overcomes chemoresistance in chronic lymphocytic leukemia. Oncogene 30: 701-713, 2011.

36. Jiao DM, Yan L, Wang LS, Hu HZ, Tang XL, Chen J, Wang J, Li Y and Chen QY: Exploration of inhibitory mechanisms of curcumin in lung cancer metastasis using a miRNA-transcription factor-target gene network. PLoS One 12: e0172470, 2017.

37. Jiang ZQ, Li MH, Qin YM, Jiang HY, Zhang X and Wu MH: Luteolininhibits tumorigenesis and induces apoptosis of non-small cell lung cancer cells via regulation of MicroRNA-34a-5p. Int J Mol Sci 19: 447, 2018.

38. Wang X, Dong K, Gao P, Long M, Lin F, Weng Y, Ouyang Y, Ren J and Zhang H: MicroRNA-34a sensitizes lung cancer cell lines to DDP treatment independent of p53 status. Cancer Biother Radiopharm 28: 45-50, 2013

39. Zhang Z, Kong Y, Yang W, Ma F, Zhang Y, Ji S, Ma EM, Liu H, Chen Y and Hua Y: Upregulation of microRNA-34a enhances the DDP sensitivity of gastric cancer cells by modulating proliferation and apoptosis via targeting MET. Oncol Rep 36: 2391-2397, 2016.

40. Xu M, Hu J, Zhou B, Zhong Y, Lin N and Xu R: TRIM29 prevents hepatocellular carcinoma progression by inhibiting Wnt/ $\beta$-catenin signaling pathway. Acta Biochim Biophys Sin (Shanghai) 51: 68-77, 2019.

41. Song X, Fu C, Yang X, Sun D, Zhang X and Zhang J: Tripartite motif-containing 29 as a novel biomarker in non-small cell lung cancer. Oncol Lett 10: 2283-2288, 2015.

42. Zhan W, Han T, Zhang C, Xie C, Gan M, Deng K, Fu M and Wang JB: TRIM59 promotes the proliferation and migration of non-small cell lung cancer cells by upregulating cell cycle related proteins. PLoS One 10: e0142596, 2015.

43. Liu C, Huang X, Hou S, Hu B and Li H: Silencing of tripartite motif (TRIM) 29 inhibits proliferation and invasion and increases chemosensitivity to cisplatin in human lung squamous cancer NCI-H520 cells. Thorac Cancer 6: 31-37, 2015.

This work is licensed under a Creative Commons Attribution-NonCommercial-NoDerivatives 4.0 International (CC BY-NC-ND 4.0) License. 